Amy Wiser, MD, FAAFP

Assistant Professor, Department of Family Medicine,

Oregon Health \& Science University, Portland, OR;

US Cervical Cancer Screening Risk-Based Management

Guidelines Research Steering, Colposcopy, and

Communication Committees
Jeffrey D. Quinlan, MD, FAAFP

Professor and Chair, Department of Family Medicine,

University of lowa Carver College of Medicine, lowa

City, IA; US Cervical Cancer Screening Risk-Based

Management Guidelines Communications Committee
Pelin Batur, MD, FACP, NCMP

Department of Subspecialty Women's Health, Ob-Gyn \& Women's Health Institute, Cleveland Clinic; Associate Professor of Ob-Gyn \& Reproductive Biology, Cleveland Clinic Lerner College of Medicine of Case Western Reserve University, Cleveland, $\mathrm{OH}$; Deputy Editor, Cleveland Clinic Journal of Medicine; US Cervical Cancer Screening Risk-Based Management Guidelines Communications Committee

\title{
Risk-based guidelines:
} Redefining management of abnormal
cervical cancer screening results

\section{ABSTRACT}

In making the 2019 guidelines for risk-based management of patients with abnormal cervical cancer screening tests and cancer precursors, the guidelines committee shifted from results-based to risk-based management recommendations, based on the patient's immediate and 5 -year risks of grade 3 or higher cervical intraepithelial neoplasia (CIN 3+). The risk is determined by current and prior screening results (human papillomavirus infection, cytology testing) and the clinical history including age. An immediate $4 \%$ or higher risk of CIN 3+ was established as the dividing line between higher and lower risks, and the corresponding management recommendations. This article reviews the changes and their evidence base and discusses clinical implications of the revised guidelines.

\section{KEY POINTS}

Management of patients with abnormal cervical cancer screening results is based on their risk of cervical cancer rather than only on the results of Papanicolaou and human papillomavirus tests.

For individuals at higher risk (ie, immediate CIN 3+ risk of $4 \%$ or higher), more frequent surveillance via colposcopy and earlier treatment is recommended.

For those at lower risk (ie, immediate CIN 3+ risk below $4 \%)$, colposcopy surveillance can be deferred.
The 2019 American Society for Colposcopy and Cervical Pathology (ASCCP) risk-based management consensus guidelines for abnormal cervical cancer screening tests and cancer precursors, published in April $2020,{ }^{1}$ represent a shift away from resultsbased management and toward risk-based management. Management of patients with abnormal cervical cancer screening results is now based on their risk of cervical cancer rather than only on results of Papanicolaou (Pap) and human papillomavirus (HPV) tests. Risk is determined based on clinical factors (including age), prior and current HPV infection (including genotyping results, if known), and cytology (Pap test) results. Calculation of risk is quite complicated and requires use of a smartphone application or a computer. ${ }^{2}$

Specifically, management is based on a patient's immediate and 5-year risks of cervical intraepithelial neoplasia grade 3 or higher $(\mathrm{CIN} 3+)$, determined by current and prior screening results as well as the clinical history, including age and past testing results. Thus, patients with the same current Pap and HPV test results may have different management recommendations based on their individual medical history.

\section{WHO WROTE THE GUIDELINES?}

A guidelines committee of 19 organizations under the direction of the ASCCP compiled the guidelines. The committee included medical societies, federal agencies, and patient advocacy organizations including the American Academy of Family Physicians, American 

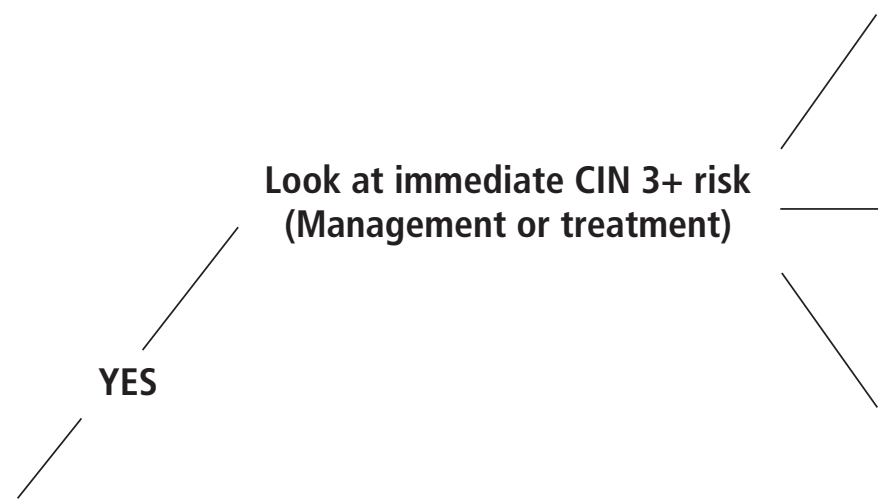

Expedited treatment

preferred

$(>60 \%)$

Expedited treatment

or colposcopy

acceptable

$(25 \%-59 \%)$

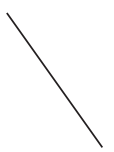

Colposcopy

recommended

$(4 \%-24 \%)$

Is immediate CIN 3+ risk $\geq 4 \%$ ?

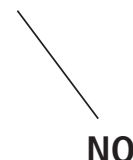

Look at 5-year CIN 3+ risk

(Surveillance)

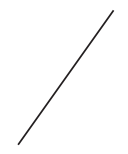

Return in 1 year

$(\geq 0.55 \%)$

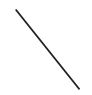

Return in 3 years

$(0.15 \%-0.54 \%)$

Return in 5 years

$(<0.15 \%)$

Figure 1. An example of patient risk evaluation.

CIN 3+ = cervical intraepithelial neoplasm grade 3 or higher

Reprinted with permission from Perkins RB, Guido RS, Castle PE, et al. 2019 ASCCP Risk-based management consensus guidelines for abnormal cervical cancer screening tests and cancer. J Low Genit Tract Dis 2020; 24(2):102-131. doi:10.1097/LGT.0000000000000525.

College of Obstetrics and Gynecology, US Centers for Disease Control and Prevention, and the National Cancer Institute.

\section{WHAT ARE THE MAIN RECOMMENDATIONS?}

The updated guidelines provide a more personalized management strategy based on the patient's CIN 3+ risk (Figure 1), using a 4\% or higher risk of CIN $3+$ as the threshold for referral for colposcopy and expedited treatment. ${ }^{1}$ For individuals at higher risk (ie, immediate CIN 3+ risk 4\% or higher), more frequent surveillance with colposcopy and earlier treatment are recommended. For those at lower risk (ie, immediate CIN 3+ risk below 4\%), surveillance with colposcopy can be deferred, and follow-up is recommended at longer intervals, ie, 1 to 5 years.

The guidelines designate CIN 3+, which encompasses CIN 3, adenocarcinoma in situ, and cervical cancer, as a cancer precursor because of the infrequent incidence of cervical cancer in the United States and because treatment of this precursor can drastically reduce cervical cancer risk. In addition, CIN 3+ provides a more consistent pathological diagnosis than cervical intraepithelial neoplasia grade 2 or higher $(\mathrm{CIN} 2+) .{ }^{1}$

Recommendations for when to conduct continued surveillance (Pap and HPV testing) remain at 1-, 3-, and 5-year intervals, consistent with the previous guidelines, ${ }^{3}$ which are 
familiar to clinicians. Additionally, the new guidelines review best practices for performing and reporting on colposcopy results, to help ensure standardization among those who perform the procedure. ${ }^{4}$

\section{TREATMENT RECOMMENDATIONS}

In patients at highest risk (ie, those with immediate CIN 3+ risk higher than 60\%), expedited treatment is recommended. ${ }^{1}$ Expedited treatment, an option in previous guidelines, is further defined, with guidance based on risk stratification. Excisional treatment is preferred to ablative therapies such as cryotherapy and laser therapy for both expedited treatment and treatment indicated by colposcopy. ${ }^{5}$ Postexcisional treatment surveillance now includes HPV-based testing for at least 25 years at 3 -year intervals. ${ }^{1}$ This recommendation is based on evidence showing that after treatment for histologic high-grade squamous intraepithelial lesions (CIN 2, CIN 3, or adenocarcinoma in situ) a patient's risk does not return to the range in the general population. ${ }^{1,6}$

\section{The goals of the new recommen- dations are to increase accuracy for treatment and to reduce complexity for providers and patients}

\section{PATHOLOGY TEST RECOMMENDATIONS}

The guidelines also recommend that laboratories should report high-grade squamous intraepithelial lesions as either CIN 2 or CIN 3 in histopathology reports, based on recommendations from the Lower Anogenital Squamous Terminology Standardization Project work group ${ }^{7}$ and the World Health Organization. ${ }^{8}$ This distinction can help with management strategies when considering the patient's reproductive goals. The concept of reproductive goals replaces the term "young women," which was used in previous guidelines. Specifically, patients with CIN 2 histopathology who also desire to maintain their fertility may be followed with close surveillance. In contrast, patients with CIN 3 histopathology should undergo excisional treatment, which could pose an increased risk of complications during a future pregnancy.

Cervical cancer screening by cytology alone is acceptable only when HPV testing is not available. ${ }^{1}$ Also acceptable is primary HPV screening (HPV alone) without cytology. The US Food and Drug Administration (FDA) has approved 2 assays for primary HPV screening - cobas HPV (Roche) ${ }^{9}$ and Onclarity HPV (Becton, Dickinson, and Company) ${ }^{10}$ - and they should be used only according to their regulatory approval. ${ }^{11}$ All positive primary HPV screening tests should include reflex triage testing (cytology) on that same specimen. ${ }^{12}$

\section{WHAT IS DIFFERENT FROM PRIOR GUIDELINES?}

The goals of the new recommendations are to increase accuracy for treatment and reduce complexity for providers and patients compared with the 2012 guidelines. The underlying concept is "equal management for equal risks" based on results of history and testing, as opposed to algorithms based on test results alone. The new recommendations introduce the concept of "clinical action thresholds," which are management scenarios that include current and past test result combinations to determine an individual's risk profile. Thresholds are based on an estimated risk of CIN 3+ at the time of the abnormal cervical cancer screening result. As noted above, an immediate risk of CIN $3+$ of $4 \%$ or higher leads to a management recommendation of colposcopy or expedited treatment. If the immediate risk is calculated as less than this $4 \%$ threshold, surveillance recommendation is then based on the specific 5-year risk of CIN $3+.{ }^{1}$

Current screening results and medical history are used to determine the CIN $3+$ risk estimate for each individual, as derived from calculated data tables. ${ }^{2}$ As further research provides nuanced understanding of the natural history of HPV and cervical carcinogenesis, it is clear that persistent HPV infection is necessary for the progression to cervical precancer and cancer. ${ }^{13-16}$ Research has also provided data on how an HPV-negative history affects the clinical meaning of current test results and an individual's risk of CIN $3+{ }^{17}$ As our understanding of cervical cancer risk and prevention continues to evolve, including the long-term impact of HPV vaccination, updated risk calculations can be more easily incorporated into guideline updates to ensure equal management for equal risks.

Another noteworthy change is guidance on the timing of surveillance after treatment 
of high-grade squamous intraepithelial lesions or higher-grade lesions. HPV screening is recommended in 6 months regardless of the status of the postexcision margin. If HPV screening is positive, referral for colposcopy is indicated. If HPV screening is negative, annual follow-up with primary HPV or cotesting for 3 years is indicated. If consecutive tests are negative, continued surveillance every 3 years for at least 25 years up to age 65 (or older if the patient is in good health), is now the standard. ${ }^{1,17}$

\section{EVIDENCE BASE FOR RISK ESTIMATES}

The formulation of risk estimates is based primarily on a Kaiser Permanente Northern California database of more than 1.5 million women who underwent routine cotesting from 2003 to 2017, including HPV genotyping for 19,000 patients. ${ }^{18}$ The data analysis was conducted at the National Cancer Institute by statisticians using updated methods to produce the risk tables. These risk estimates and tables underlie the guidelines. ${ }^{17}$

These data are the largest and the longest real-world clinical experience with HPVbased screening and provide risk-based evidence for most of the common decision points of screening. Although the database has been criticized for limited inclusion of diverse socioeconomic cohorts in the context of a population with cervical cancer risk that is lower than the national average, comparison of risks and risk-based management to that of other large cohorts or clinical trials has validated the data used in the guidelines. ${ }^{1,6,18,19}$ The updated guidelines accommodate the 3 cervical screening strategies available in the United States: primary HPV screening, cotesting with HPV testing and cervical cytology, and cervical cytology alone. . $^{1,20}$

\section{WHAT IS THE EXPECTED CLINICAL IMPACT?}

The new guidelines provide a framework for triaging high-risk individuals to treatment while avoiding unnecessary procedures and tests for lower-risk individuals, thus achieving a better balance of benefits to harm for cervical cancer screening. It is estimated that the number of patients referred for colposcopy will be reduced from $9.8 \%$ to $8.3 \%$ over 2 rounds of screening with the transition to risk-based vs results-based interventions. ${ }^{1}$

The guidelines are designed to be enduring. Integral to the data and risk-estimate analysis is the ability to include new technologies. When a new screening strategy is devised or a new test is FDA-approved, it can be considered for inclusion in the guidelines, assuming that sufficient data are available. Likewise, as HPV vaccinations decrease the prevalence of HPV infections and the risk of cervical carcinoma, management recommendations will incorporate these data. It is hoped that a longer interval will transpire before the next guideline revision is needed, leading to more stable clinical management for providers.

\section{HOW WILL THIS CHANGE DAILY PRACTICE?}

Because of the complexities involved in calculating the CIN 3+ risk estimates for each patient, it will be nearly impossible for clinicians to memorize the algorithms. Risk estimations now require use of computerized technology. The extensive risk table compiled by the $\mathrm{Na}$ tional Cancer Institute is accessible on the National Institutes of Health website, https:// CervixCa.nlm.nih.gov/RiskTables. ${ }^{2}$ A smartphone application that calculates the risk is available for purchase from the ASCCP. ${ }^{21} \mathrm{Cli}$ nicians should acquaint themselves with use of the risk calculator, which will allow them to competently guide the management of this patient population.

Most scenarios commonly encountered in clinical practice can be easily managed by calculating the risk estimates and applying them to the guidelines. The detailed management protocols will allow primary care providers who do or do not manage abnormal cervical cancer screening tests to be able to accurately guide patient management and avoid unnecessary referrals or procedures. In settings where resources are limited, the protocols will enable clinicians to confidently refer for treatment patients who are at high risk and would benefit from immediate treatment.

\section{WHEN DO THE GUIDELINES NOT APPLY?}

Guidelines apply to average-risk, asymptomatic individuals with an intact cervix, based on screening management data for patients
The guidelines provide a framework for triaging high-risk individuals to treatment while avoiding unnecessary procedures and tests for those at lower risk 
ages 25 to 65 . The guidelines separately address management of special populations, ie, those who are under age 25 or over age 65 , are pregnant, are receiving immunosuppressive therapy, or have had a hysterectomy. ${ }^{1}$ Notably, an accurate risk estimation for patients under age 25 is challenging. This is due primarily to a changing influence of HPV vaccination on cervical cancer risks for this population, as well as a higher rate of regression of histologic high-grade dysplasia and a lower incidence and progression risk of invasive cervical cancer. Pregnancy necessitates management and treatment options that consider the risk of a missed cancer diagnosis to both the fetus and the patient.
Also, some cytologic and HPV results have been found to be disproportionately important for the risk of invasive cancer. Specifically, results showing the genotype HPV 18, HPV-negative atypical glandular cells, and atypical squamous cells cannot exclude highgrade atypical squamous cells. These patients are recommended for colposcopy even though they do not meet the $4 \%$ immediate CIN 3+ risk threshold. For safety reasons, this recommendation considers absolute risk of cancer in addition to risk of precancer. ${ }^{1}$

\section{DISCLOSURES}

The authors report no relevant financial relationships which, in the context of their contributions, could be perceived as a potential conflict of interest.

\section{REFERENCES}

1. Perkins RB, Guido RS, Castle PE, et al. 2019 ASCCP Risk-based management consensus guidelines for abnormal cervical cancer screening tests and cancer. J Low Genit Tract Dis 2020; 24(2):102-131. doi:10.1097/LGT.0000000000000525

2. National Institutes of Health. Risk estimates supporting the 2019 ASCCP risk-based management consensus guidelines. https://CervixCa.nlm.nih.gov/RiskTables. Accessed August 31, 2021.

3. Massad LS, Einstein MH, Huh WK, et al. 2012 updated consensus guidelines for the management of abnormal cervical cancer screening tests and cancer precursors. Obstet Gynecol 2013; 121(4):829846. doi: 10.1097/AOG.0b013e3182883a34

4. Khan MJ, Werner CL, Darragh TM, et al. ASCCP Colposcopy standards: role of colposcopy, benefits, potential harms, and terminology for colposcopic practice. J Low Genit Tract Dis 2017; 21(4):223229. doi:10.1097/LGT.0000000000000338

5. D'Alessandro P, Arduino B, Borgo M, et al. Loop electrosurgical excision procedure versus cryotherapy in the treatment of cervical intraepithelial neoplasia: a systematic review and meta-analysis of randomized controlled trials. Gynecol Minim Invasive Ther 2018; 7(4):145-151. doi:10.4103/GMIT.GMIT_56_18

6. Clarke MA, Unger ER, Zuna R, et al. A systematic review of tests for postcolposcopy and posttreatment surveillance. J Low Genit Tract Dis 2020; 24(2):148-156. doi:10.1097/LGT.0000000000000526

7. Darragh TM, Colgan TJ, Cox JT, et al; Members of LAST Project Work Groups. The Lower Anogenital Squamous Terminology Standardization Project for HPV-Associated Lesions: background and consensus recommendations from the College of American Pathologists and the American Society for Colposcopy and Cervical Pathology. Arch Pathol Lab Med 2012; 136(10):1266-1297.

doi:10.5858/arpa.LGT200570

8. World Health Organization. WHO guidelines for screening and treatment of precancerous lesions for cervical cancer prevention. Geneva, Switzerland: World Health Organization; 2013. https://www.ncbi.nlm.nih.gov/books/NBK195239/pdf/Bookshelf NBK195239.pdf. Accessed August 31, 2021.

9. Cobas PI. https://www.accessdata.fda.gov/cdrh_docs/pdf10/ p100020s017c.pdf. Accessed August 31, 2021.

10. Onclarity HPV assay package insert. https://www.accessdata.fda. gov/cdrh_docs/pdf16/P160037C.pdf. Accessed August 31, 2021.

11. FDA executive summary. New approaches in the evaluation for high-risk human papillomavirus nucleic acid detection devices. https://www.fda.gov/media/122799/download. Accessed August 31, 2021.
12. Huh WK, Ault KA, Chelmow D, et al. Use of primary high-risk human papillomavirus testing for cervical cancer screening: interim clinical guidance. Gynecol Oncol 2015; 136(2):178-182. doi:10.1016/j.ygyno.2014.12.022

13. McCredie MR, Sharples KJ, Paul C, et al. Natural history of cervical neoplasia and risk of invasive cancer in women with cervical intraepithelial neoplasia 3: a retrospective cohort study. Lancet Oncol 2008; 9(5):425-434. doi:10.1016/S1470-2045(08)70103-7

14. Rodríguez AC, Schiffman M, Herrero R, et al. Rapid clearance of human papillomavirus and implications for clinical focus on persistent infections. J Natl Cancer Inst 2008; 100(7):513-517. doi:10.1093/jnci/djn044

15. Chen Z, Schiffman M, Herrero R, et al. Classification and evolution of human papillomavirus genome variants: Alpha-5 (HPV26, 51, 69, 82), Alpha-6 (HPV30, 53, 56, 66), Alpha-11 (HPV34, 73), Alpha-13 (HPV54) and Alpha-3 (HPV61). Virology 2018; 516:86-101. doi:10.1016/j.virol.2018.01.002

16. Demarco M, Egemen D, Raine-Bennett TR, et al. A study of partial human papillomavirus genotyping in support of the 2019 ASCCP Risk-Based Management Consensus Guidelines. J Low Genit Tract Dis 2020; 24(2):144-147. doi:10.1097/LGT.0000000000000530

17. Egemen D, Cheung LC, Chen X, et al. Risk estimates supporting the 2019 ASCCP Risk-Based Management Consensus guidelines. J Low Genit Tract Dis 2020; 24(2):132-143. doi:10.1097/LGT.0000000000000529

18. Cheung LC, Egemen D, Chen X, et al. 2019 ASCCP Risk-based management consensus guidelines: methods for risk estimation, recommended management, and validation. J Low Genit Tract Dis 2020; 24(2):90-101. doi:10.1097/LGT.0000000000000528

19. Gage JC, Hunt WC, Schiffman M, et al. Similar risk patterns after cervical screening in two large US populations: implications for clinical guidelines. Obstet Gynecol 2016; 128(6):1248-1257. doi:10.1097/AOG.0000000000001721

20. Schiffman M, Wentzensen N, Perkins RB, Guido RS. An Introduction to the 2019 ASCCP risk-based management consensus guidelines. J Low Genit Tract Dis 2020; 24(2):87-89. doi:10.1097/LGT.0000000000000531

21. American Society for Colposcopy and Cervical Pathology. ASCCP risk-based management consensus guidelines. https://www.asccp. org/mobile-app. Accessed August 31, 2021.

Address: Amy Wiser, MD, FAAFP, Emma Jones Hall, Oregon Health \& Science University, 3181 SW Sam Jackson Park Rd, Portland, OR 97239; wisera@ohsu.edu 